Gut, 1976, 17, 652-654

\title{
An apparatus for the anaerobic ultrafiltration of bile and other biological fluids
}

\author{
LYNETTE I. WILKIE, G. H. CARTER, V. A. HALLEWELL, AND \\ D. JUNE SUTOR ${ }^{1}$
}

From the Department of Chemistry, University College, London

SUMMARY A simple apparatus is described for the anaerobic ultrafiltration of a few millilitres of bile or other biological fluids. The principle employed enables viscous liquids which would normally block the membrane to be ultrafiltered successfully.

Ultrafiltration selectively separates molecules according to size by employing membranes with pore diameters usually in the range 0.001 to $0.05 \mu \mathrm{m}$. These retain solutes of larger dimensions and allow solvent and solutes of smaller dimensions to pass. Compressed gas or another source of pressure is necessary to force the solvent and solute through the membrane. Ultrafiltration of plasma has been used extensively to help in the differentiation of bound, complexed, and ionized calcium, and simple techniques have been described for this purpose (Varghese, 1973).

In our investigation into the composition of bile, similar studies were necessary to determine whether certain ions are bound to macromolecules such as the micelles and mucoprotein. As in the case of plasma, only a few millilitres of bile are generally available for making measurements, and these must also be ultrafiltered anaerobically-that is, with no air space in contact with the fluid-so that bicarbonate is not lost and the $\mathrm{pH}$ stays constant. However, unlike plasma, bile is often a viscous liquid which soon clogs the membrane resulting in only a small volume of filtrate and concentration polarisation at the membrane surface. Concentration polarisation refers to the concentration gradient above the membrane with the macrosolute concentration greatest at the surface. It restricts passage through the filter and, in effect, acts as a secondary membrane. When our work on bile was started, we could not find a commercially-available, inexpensive ultrafiltration apparatus which would overcome these problems, and it was therefore necessary to design one.

The bile or sample to be ultrafiltered is collected

${ }^{1}$ Address for correspondence: Dr D. J. Sutor, Department of Chemistry, University College, Gower Street, London WC1.

Received for publication 14 May 1976 anaerobically in a $10 \mathrm{ml}$ Gillette plastic syringe with a Luer fitting. It is then filtered anaerobically through a prefilter and Millipore filter (pore size, $0.45 \mu \mathrm{m})$ to remove any precipitate and soluble and insoluble mucus of high molecular weight. A Millipore stainless steel microsyringe filter-holder with the dead space filled in can be used for this purpose. All biles, except those which cannot be drawn into a hypodermic needle (internal diameter, $0.6 \mathrm{~mm}$ ) because they are too viscous, can be forced through the filters by hand pressure. The very viscous biles cannot be filtered, but such samples are not usually used in quantitative bile studies because accurate aliquots cannot be measured. The technique used in the anaerobic filtration of bile through a Millipore filter consists of attaching a small length of silicone rubber tubing to the outlet of the filter holder and filling this with filtered bile by depressing the plunger of the syringe. The first $50 \mu \mathrm{l}$ are discarded because these are unlikely to be anaerobic and the tubing is then connected to another $10 \mathrm{ml}$ Gillette syringe with the plunger at zero. Filtered bile is forced into this syringe and the small air bubble (from the air in the Luer fitting) is immediately expelled.

The ultrafiltration cell, which is made of stainless steel, is shown expanded in Fig. 1. There are two main parts, the top and the base. The base has an internal diameter of $2.63 \mathrm{~cm}$ designed to take an ultrafilter of $25 \mathrm{~mm}$ diameter. The type of ultrafilter used depends on the molecular separation required. Both gallbladder and hepatic bile which have been filtered as above, can be ultrafiltered through membranes with a nominal molecular weight cut-off of 10000 or more (Amicon ultrafilters, type PM). The filter lies on a photo-etched stainless steel support screen in which the hole diameter is 100-150 $\mu \mathrm{m}$ (available from Millipore Ltd.). The screen 

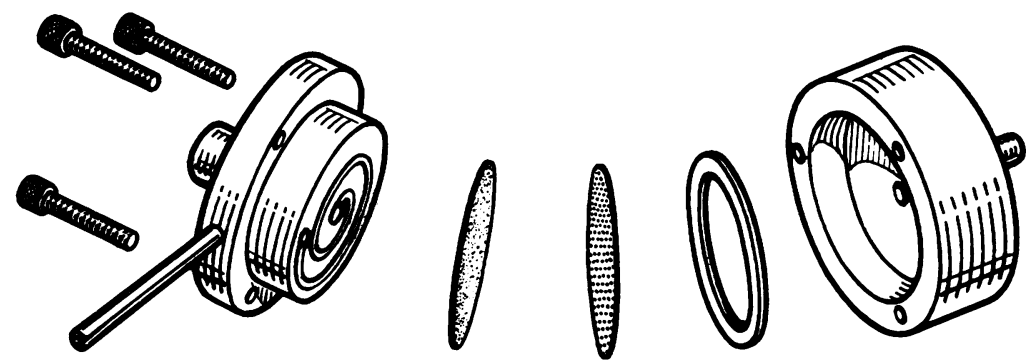

Fig. 1 Expanded drawing of ultrafiltration cell. From right to left the parts are the base, Teflon $O$-ring, support screen, ultrafilter, the top, and screws. The spiral groove with inlet and outlet holes can be seen in the top part. The groove is $1.5 \mathrm{~mm}$ wide, $0.25 \mathrm{~mm}$ deep, and its cross-section is the arc of a circle of radius $1.25 \mathrm{~mm}$. The constrictor valve is shown in the assembled apparatus in Fig. 2.

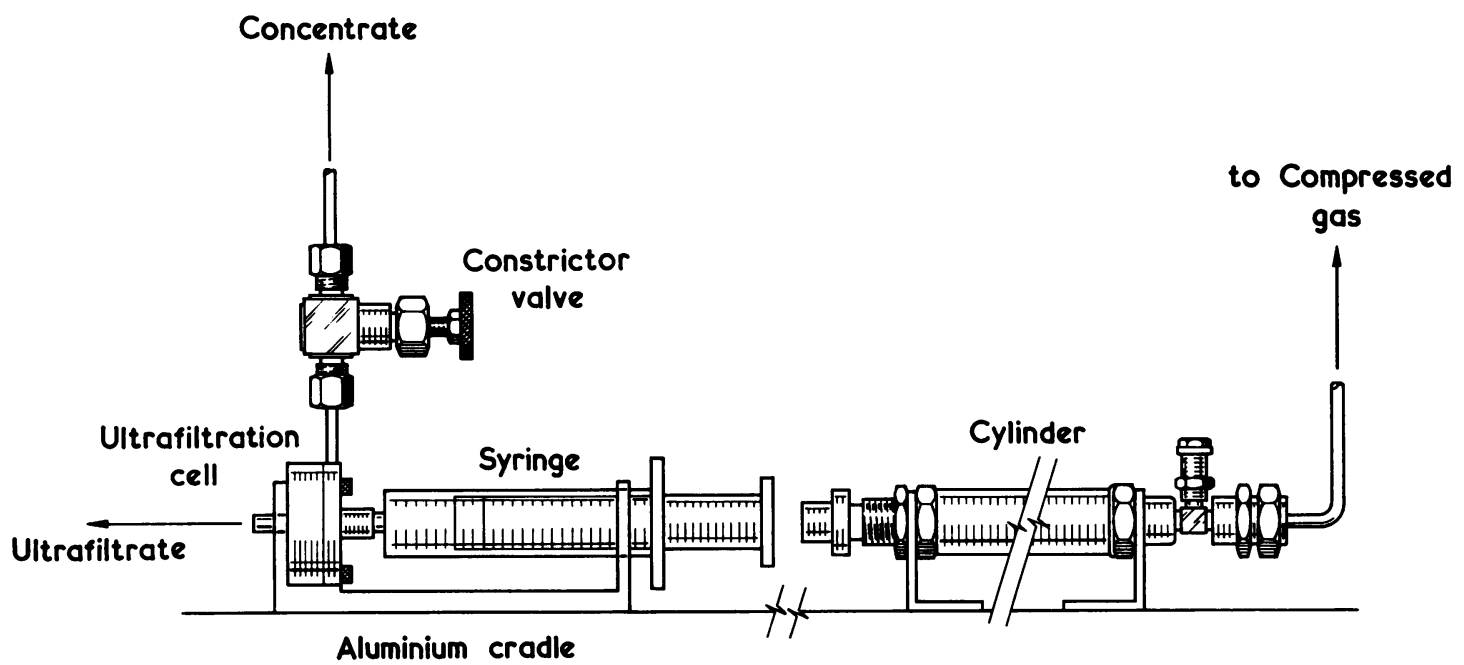

Fig. 2 Diagram of the ultrafiltration scheme showing the assembled cell, constrictor valve, syringe with sample, and cylinder. The cylinder and the space between the plunger and piston rod have been compressed to save space.

rests on a flat Teflon O-ring (Millipore Ltd.) which fits into the flat base of the cell. The centrallylocated hole in the base provides the exit for the ultrafiltrate which is collected in silicone rubber tubing attached to the outlet. A microsyringe can be used to withdraw the ultrafiltrate through the wall of the tubing.

The top and base fit together exactly without allowance for the O-ring, support screen, and ultrafilter. The top is thus in firm contact with the ultrafilter and is held in position by three screws. If the ultrafilter is covered with a Millipore filter (pore size, $0.45 \mu \mathrm{m}$ ), it will not stick to the top and can be reused. The top has a central Luer inlet into which the syringe containing the sample fits. During ultrafiltration, the sample is forced through the inlet to the membrane and then over the membrane through the thin spiral groove, thus minimising concentration polarisation. The concentrated sample passes up and out through the side of the cell into an arm with a constrictor valve attached. This valve provides back pressure for the system and the one used is a stainless steel needle valve suitable for high pressures (F. J. Hone and Co., London, type 43002). Positive control and shut-off are obtained by gently turning the knob in Fig. 2. Ultrafiltration is started with the valve closed, but, once the degree of concentration required has been reached or 
ultrafiltration has virtually stopped, the valve is opened gently so that fresh bile moves into the thin channel and then closed again.

The assembled cell and syringe containing the sample are supported in an aluminium cradle screwed to a base to which a cylinder is also attached. A diagram of the set-up is given in Fig. 2. The cylinder is a standard duty, single acting pneumatic one with a bore size of $1.905 \mathrm{~cm}$ and a stroke of 7.62 cm (Enots Ltd., England). The piston rod which extends from the cylinder and applies the pressure to the syringe has a small piece of Teflon added. This has been machined to fit into the open end of the plunger of a $10 \mathrm{ml}$ Gillette syringe. The cylinder is attached by nylon hose to a standard pressure regulator gauge on a cylinder of compressed nitrogen. Ultrafiltration usually proceeds satisfactorily at an applied pressure to the syringe of $20 \mathrm{psi}$. Our equipment can be enclosed in a heated box so that Millipore filtration and ultrafiltration can be carried out at $37^{\circ} \mathrm{C}$. Under these conditions, the ends of the outlet tubing for the ultrafiltrate and concentrate are immersed in water to minimise evaporation.

We thank Mr J. Cresswell for drawing the diagrams and the Medical Research Council for financial assistance.

\section{Reference}

Varghese, Z. (1973). Determination of plasma calcium fractions. Annals of Clinical Biochemistry, 10, 120-124. 